\title{
Mort du colonel J. Thomann
}

Mlle de la Beausserie, secrétaire à la Direction de la Croix-Rouge française, Paris ; Mne de Rolland, secrétaire de la Croix-Rouge française, Toulouse; M. André Fournel, journaliste; M. et $\mathbf{M}^{\text {me }}$ de Schulthess ; M. Hegner ; M. Keller ; $\mathbf{M}^{\text {me }}$ Tsaldyris, de la Croix-Rouge hellénique; $\boldsymbol{M}$. et $\mathbf{M}^{\mathrm{me}}$ Ritchard, de la CroixRouge suédoise ; M11e Duvillard, de la Ligue des Sociétés de la Croix-Rouge; M11e Petchnik, Caritas Verband, Allemagne; M. Hammarskjöld, greffier de la Cour de justice internationale, envoyé extraordinaire et ministre plénipotentiaire en disponibilité, Institut de droit international, Suède; $M$. et $\mathbf{M}^{\text {me }}$ Jean Masure, de l'Administration centrale de la Croix-Rouge de Belgique, Bruxelles; Miss et Mr. Walker, Londres; Mrs. Mac Phael, Canadian Red Cross; Mr. Bozic (Yougoslavie); un groupe d'ex-prisonniers et déportés français hospitalisés au Sanatorium de Praz-Coutant (Haute-Savoie); Mne Gubler, de la Croix-Rouge chilienne; M. Sidzikauskas (Lithuanie); S. Exc. M. Ferenc Gordon, envoyé extraordinaire et ministre plénipotentiaire de Hongrie à Berne; Miss Cochrane, du Conseil international des infirmières à Londres; Lady Iman et son fils ; Miss Bennett, de la Croix-Rouge britannique, accompagnée de sa sœur; M. J.J.-G. de Rueda, délégué de la Croix-Rouge mexicaine; M. R.F. Bennewitz, (Buenos-Ayres).

\section{iMort du colonel J. Thomann}

Le Comité international de la Croix-Rouge a appris avec une profonde tristesse la mort subite du colonel J. Thomann, ancien pharmacien-chef de l'armée suisse, qui durant bien des années collabora avec dévouement à son œuvre traditionnelle.

Bourgeois de St-Gall, où il naquit en $\mathrm{r} 872, \mathrm{M}$. Thomann fit des études de pharmacie à l'Université de Berne, études qu'il poursuivit jusqu'au doctorat, à Genève, puis à l'Ecole polytechnique de Zurich. 


\section{Mort du colonel J. Thomann}

En IgII il devint officier instructeur des troupes sanitaires et pharmacien-chef de l'armée ; il fut promu au grade de colonel en 1923.

Expert de la Confédération pour toutes les questions qui relèvent du Service de santé de l'armée, il représenta souvent la Suisse dans de nombreux congrès à l'étranger.

Dès l'année I933, le colonel Thomann donna des conférences et des cours à la Faculté de pharmacie de l'Université de Berne où sa haute compétence technique était particulièrement appréciée.

C'est en 1927 que le colonel Thomann fut appelé à faire partie de la Commission que le Comité international avait été chargé de constituer, par la XII ${ }^{\theta}$ Conférence des Sociétés nationales de la Croix-Rouge, pour étudier, du point de vue international, le problème de la standardisation du matériel sanitaire aux fins de contribuer à son perfectionnement.

Il prit une part très active et prépondérante aux travaux de la Commission et il lui apporta une aide précieuse en présentant d'importants rapports techniques: sur le paquet de pansement au vioforme, la formation et l'instruction des équipes de désinfection et leur matériel, sur les plaques d'identité, sur les moyens de transport de blessés et de malades en montagne, etc.

En 1940, le colonel Thomann renonça à ses hautes fonctions de pharmacien-chef de l'armée suisse. Toutefois, il ne cessa de rendre encore de précieux services et déploya, jusqu'à la dernière heure de sa vie, une grande activité scientifique; il collabora notamment, et de la façon la plus constante à la Revue internationale de la Croix-Rouge en composant de très nombreuses notices bibliographiques où il mettait les lecteurs au courant de la vie scientifique médicale avec une grande curiosité d'esprit.

La Revue internationale s'associe de tout cœur à l'hommage ému qui a été rendu à la mémoire du colonel Thomann, par tous ceux qui ont pu apprécier le sens de l'humour, la vivacité des réparties, les dons de sociabilité de ce travailleur de l'esprit, fidèle et consciencieux. 


\section{Notes et documents}

Le Comité international n'a pas manqué de témoigner à la famille du colonel Thomann l'expression de sa profonde sympathie et il gardera un souvenir reconnaissant de celui qui servit avec tant de dévouement le grand idéal de la CroixRouge.

L. D.

\section{NOTES ET DOCUMENTS}

\section{Mémorandum du Comité international de la Croix-Rouge sur la situation actuelle des prisonniers de guerre ${ }^{2}$}

Gendve, le 2 r août r945.

$\mathrm{Au}$ moment où les Puissances alliées fixent d'un commun accord certaines des dispositions qu'elles prendront consécutivement à la cessation de la guerre en Europe, le Comité international de la Croix-Rouge ne saurait se désintéresser de la situation présente et future de nombreux prisonniers de guerre et internés civils.

En effet, les hostilités entre les Puissances alliées et le Reich allemand ont pris fin non par un traité, mais par la capitulation inconditionnelle des forces militaires allemandes, avec la disparition du Gouvernement allemand. Dès lors, les prisonniers de guerre allemands se trouvent dans une situation nouvelle.

Cette situation ne peut pas être réglée actuellement par le moyen d'un accord ad hoc auquel seraient parties les Puissances détentrices et l'Etat auquel ressortissent les prisonniers de guerre en question. C'est pourquoi le Comité international de la CroixRouge - sans avoir à se prononcer sur la situation ainsi créée estime que les prisonniers de guerre doivent continuer à bénéficier des garanties que leur assurent les Conventions existantes et que ces conventions conservent toute leur portée, même si

${ }^{1}$ Ce mémorandum a été adressé aux Gouvernements américain, belge, britannique, français, soviétique ainsi qu'à la Commission de contrôle interallié en Allemagne. 